\title{
Multidisciplinary collaboration in gallbladder carcinoma treatment: A case report and literature review
}

\author{
ZHENG-YUN ZOU ${ }^{1}$, JING YAN $^{1}$, YU-ZHENG ZHUGE $^{2},{\text { JUN } \text { CHEN }^{3}, \text { XIAO-PING QIAN }^{1} \text { and BAO-RUI LIU }}^{1}$ \\ ${ }^{1}$ Comprehensive Cancer Center, Drum-Tower Hospital, Medical School of Nanjing University, \\ Clinical Cancer Institute of Nanjing University; ${ }^{2}$ Gastrointestinal Endoscopy Center, Drum-Tower Hospital, \\ Medical School of Nanjing University; ${ }^{3}$ Department of Pathology, Drum-Tower Hospital, \\ Medical School of Nanjing University, Nanjing, Jiangsu 210008, P.R. China
}

Received April 11, 2015; Accepted May 23, 2016

DOI: $10.3892 / \mathrm{ol} .2016 .5020$

\begin{abstract}
Gallbladder carcinoma (GBC) is a rare and highly aggressive disease. The diagnosis of this cancer is difficult due to its occult onset. Hence, GBC is often detected late and at an advanced stage. Although physicians and researchers are continually working to improve the treatment for advanced-stage disease, GBC is usually associated with short survival times. The present study describes a case of GBC that was first diagnosed with accompanying cholecystolithiasis at the time of cholecystectomy. Cancer relapse occurred 1.5 years after the cholecystectomy. Multidisciplinary collaboration was involved in the decision-making process for the treatment of this aggressive recurrence, and the survival time was successfully extended to 26 months. Importantly, high-grade intraepithelial neoplasia and positive margins had previously been detected post-cholecystectomy at a different institution, but were ignored. Relapse may have been preventable had the cancer been diagnosed when it was initially observed. Taken together, these findings suggest that multidisciplinary collaboration should be considered for the management of advanced GBC, whereby patients will benefit from improved survival times. Furthermore, it is recommended that samples obtained from patients undergoing cholecystectomy should more carefully analyzed for evidence of cancerous or precancerous tissues.
\end{abstract}

\section{Introduction}

Gallbladder carcinoma (GBC) is rarely observed in Europe and the United States; however, the incidence and mortality rates in Asian countries, including China, Japan and Korea, are much

Correspondence to: Dr Bao-Rui Liu, Comprehensive Cancer Center, Drum-Tower Hospital, Medical School of Nanjing University, Clinical Cancer Institute of Nanjing University, 321 Zhongshan Road, Nanjing, Jiangsu 210008, P.R. China

E-mail: baoruiliu@nju.edu.cn; baoruiliu07@163.com

Key words: gallbladder carcinoma, multidisciplinary collaboration, cancerous or precancerous tissue, survival times, cholecystectomy higher (5.2/100,000, 4/100,000 and 5.6/100,000 individuals, respectively) (1). The majority of patients with GBCs are diagnosed at an advanced stage, which is due, in part, to the aggressive nature of the tumor and its rapid progression. Once the cancer has reached an advanced stage, curative surgical resection can no longer be performed (2), leaving any GBC patients with a poor prognosis; the median overall survival time has been reported at only 8.2 months and the 1-year survival rate for patients with stage IV disease is estimated at $1 \%(3,4)$.

The current diagnostic methods for GBC include medical imaging and bile cytological analysis. Combination of the two modalities has been shown to facilitate improved rates of diagnosis (5), but the accuracy and utility of each of these strategies are limited by their reliance on a physician's subjective evaluation and how the sampled tissue is handled prior to testing. Thus, accurate and early diagnosis remains a significant clinical challenge. Furthermore, GBC usually presents with an occult onset, therefore malignancies are commonly detected incidentally during cholecystectomy for benign diseases (6). Neglecting to further pursue these incidental findings may lead to an undiagnosed GBC, thus allowing the cancer to become more advanced and life-threatening.

The treatment options for late-stage GBC treatment include enrolling in a clinical trial, gemcitabine- or fluoropyrimidine-based chemotherapy, and/or supportive care, all of which provide only palliative relief for GBC patients (7). The specific dose, length of treatment or combination regimens of chemotherapy are not included in the National Comprehensive Cancer Network (NCCN) guidelines (7). Furthermore, the use of chemoradiation, targeted therapies, and immunotherapy remain controversial. The optimal comprehensive treatment for GBC patients has yet to be determined. The current study presents a case of GBC that should have been detected at an early stage at a different institution 2 years beforehand. The advanced GBC was then managed by a multidisciplinary collaboration, whereby the survival time of the patient was extended to 26 months.

\section{Case report}

A 62-year-old male with a 1.5-year history of cholelithiasis and cholecystectomy was admitted to the Cancer Centre of 

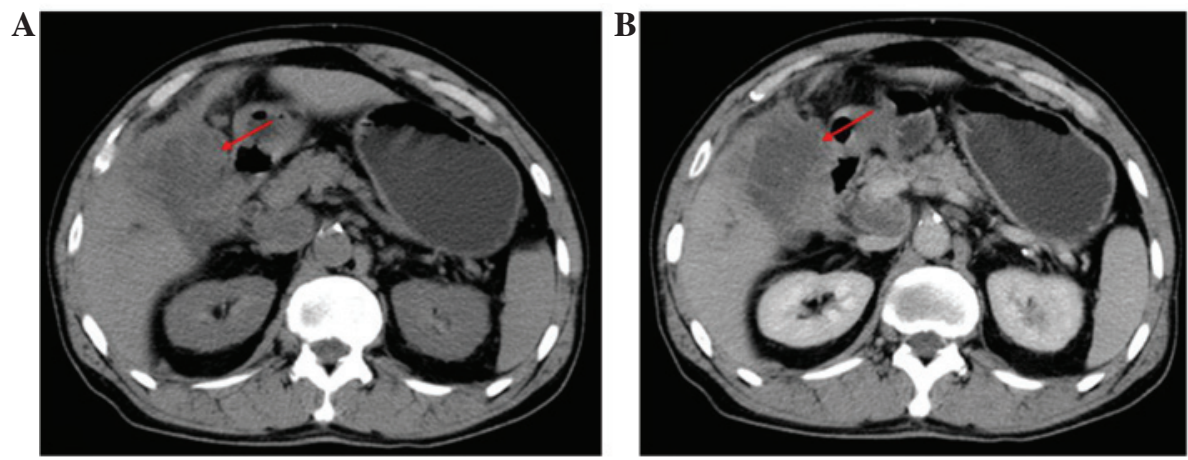

Figure 1. (A) Abdominal CT scan prior to exploratory laparotomy showing a mass (red arrow) located in the gallbladder area, with ill-defined boundaries, indicating possible malignancy and visceral invasion. (B) Abdominal CT scan with contrast enhancement. CT, computed tomography.

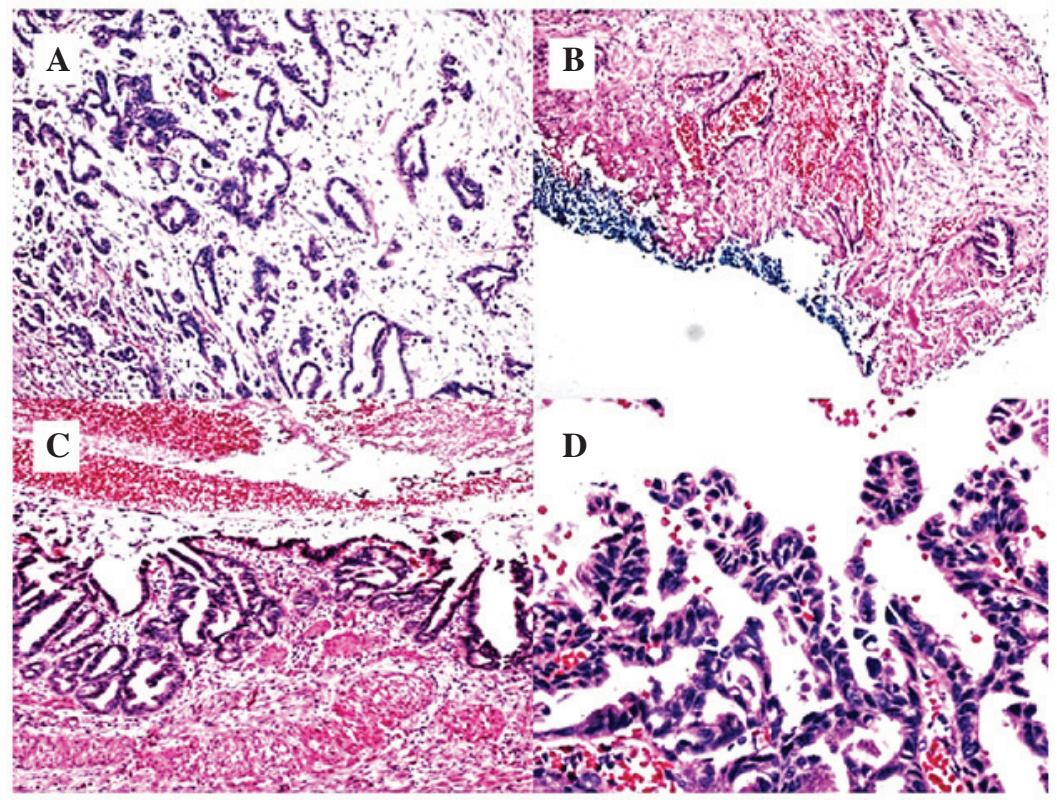

Figure 2. (A) Hematoxylin and eosin (HE) staining of excised connective tissue from the gastrocolic ligament revealing metastatic invasive adenocarcinoma of the gallbladder (magnification, x10). (B) HE staining showing intraepithelial neoplasia (IN) along the specimen margin and the neck of the gallbladder (magnification, $\mathrm{x} 4$ ). (C and D) Pathological review (HE stain) of the paraffin slides following cholecystectomy demonstrating chronic cholecystitis and extensive IN with an invasive growth pattern [magnification, (C) x10 and (D) x40].

Nanjing Drum Tower Hospital (Nanjing, Jiangsu, China) in February 2011, due to a dull pain in the right upper quadrant of the abdomen for the previous 30 days. Computed tomography $(\mathrm{CT})$ scan results (Fig. $1 \mathrm{~A}$ and $\mathrm{B}$ ) demonstrated a mass located on the gallbladder and cancer invasion of the surrounding tissues. Multiple abdominal and retroperitoneal lymph node metastases, implantation metastases, and dilation of the intrahepatic and extrahepatic bile ducts were also detected. Laboratory testing indicated normal liver function; however, the blood levels of carcinoembryonic antigen (CEA; $40.9 \mathrm{ng} / \mathrm{ml}$; normal range, $0-10 \mathrm{mg} / \mathrm{ml}$ ) and cancer antigen 19-9 (CA19-9; $378 \mathrm{U} / \mathrm{ml}$; normal range, 0-30 U/ml) were elevated. An exploratory laparotomy was performed at 6 days after the CT scan, and a suspicious mass was resected from the gastrocolic ligament. Hematoxylin and eosin (HE) staining of excised connective tissue from the gastrocolic ligament showed cells that were poorly differentiated, with a loss of polarity, an increased nucleoplasm ratio and nuclear fission and an incomplete glandular cavity with mucus, all of which indicated metastatic invasive adenocarcinoma of the gallbladder (Fig. 2A). The healthcare team decided that the risk benefit profiles of any potential treatment options were unacceptable in light of the late stage of the disease and the mild clinical manifestation. Previously prepared HE-stained paraffin-embedded sections (thickness, $5 \mu \mathrm{m}$ ) obtained from a cholecystectomy performed at the Department of General Surgery (Nanjing Drum Tower Hospital, Nanjing, Jiangsu, China) 2 years previously were subjected to a histopathological review, and chronic cholecystitis and extensive intraepithelial neoplasia (IN) with an invasive growth pattern were observed (Fig. 2B-D).

At a follow-up examination performed 3 months after the laparotomy, the disease was found to have progressed. The patient presented with a variety of disease-related symptoms, including icteric sclera, xanthochromia, yellowish discoloration of the urine, light-colored excrement and generalized pruritus. Laboratory tests revealed markedly elevated levels of total bilirubin (TBI; $255.8 \mu \mathrm{mol} / 1$; normal range, 5-20.5 $\mu \mathrm{mol} / \mathrm{l}$ ) 


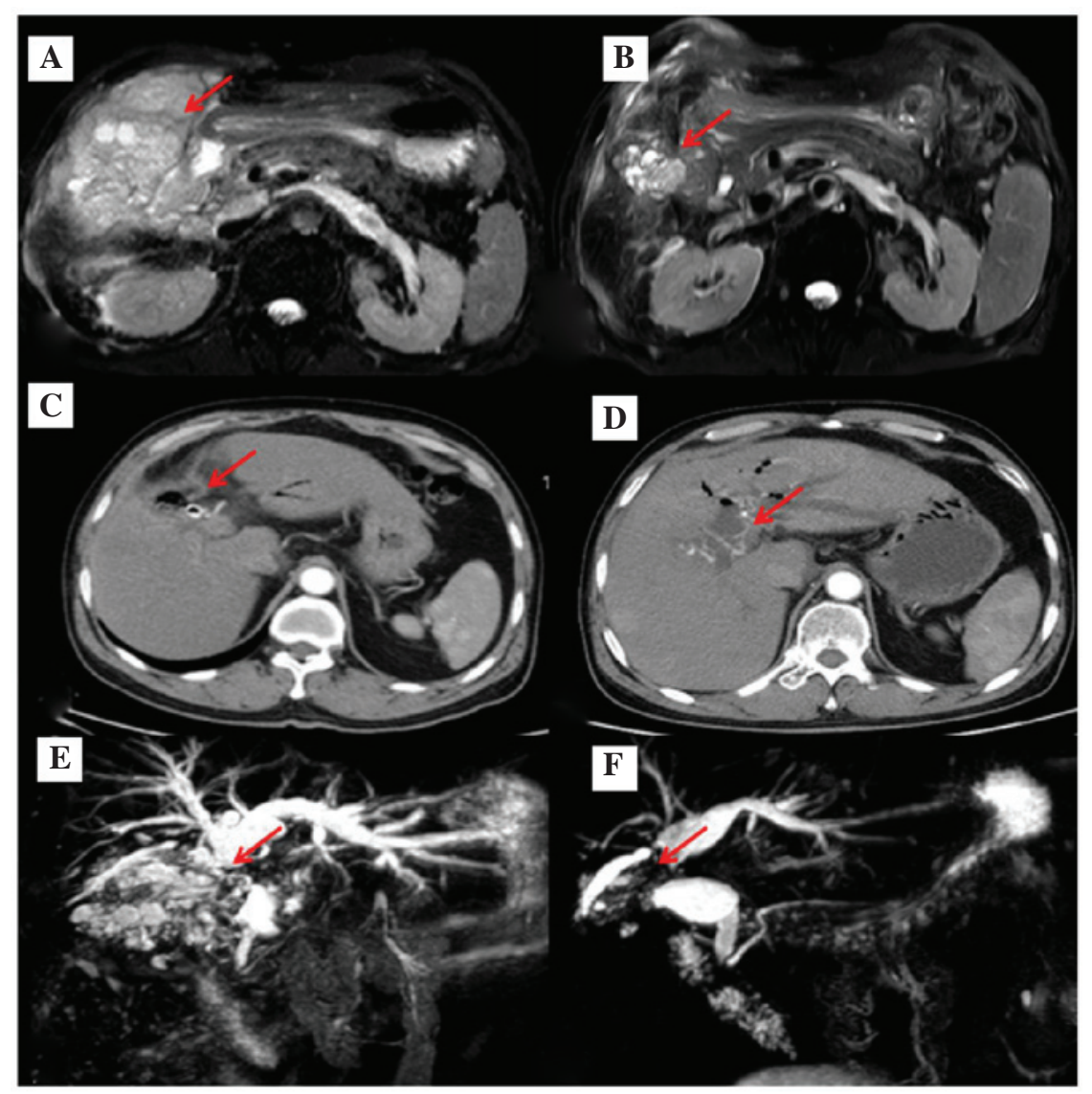

Figure 3. (A) MR imaging showing a mass (red arrow) located in the gallbladder area (T2) prior to the start of treatment. MR imaging and computed tomography scans at (B) 8, (C) 64 and (D) 84 weeks post-treatment, showing a gradually shrinking mass (red arrows). (E) MRCP prior to chemotherapy revealing dilation of the intrahepatic bile duct (red arrow). (F) MRCP demonstrating the intrahepatic bile duct (red arrow) decreasing in size 31 weeks after the start of chemotherapy. MR, magnetic resonance; MRCP, MR cholangiopancreatography.

and direct bilirubin (DBI; $161.3 \mu \mathrm{mol} / 1 ; 1.7-6.8 \mu \mathrm{mol} / \mathrm{l})$. Moreover, the blood levels of tumor markers CEA $(87.5 \mathrm{ng} / \mathrm{ml})$ and CA19-9 (933.3 U/ml) were also increased. Examination by magnetic resonance cholangiopancreatography indicated the recurrence of GBC with invasion of the liver, its surrounding peritoneum and the hilar area, as well as retroperitoneal lymph node metastasis (Fig. 3A and E). The intrahepatic bile duct was also observed to be dilated. Gene mutation analysis was performed on the tumor cells to investigate the KRAS proto-oncogene, GTPase (KRAS) gene, which is frequently mutated in cholangiocarcinoma, and indicated that the gene was the wild-type. Tumor-node-metastasis staging of the tumor, according to the American Joint Committee on Cancer (AJCC) grading system for gallbladder cancer (7th edition, 2010) (8), provided a classification of T4N2M1.

The patient underwent multiple treatments of various modalities, including percutaneous transhepatic cholangial drainage (PTCD), chemotherapy, chemoradiation, targeted therapy and immunotherapy. The strategy consisted of first delivering 12 cycles of chemotherapy, each using a 2 -week schedule of gemcitabine $(1 \mathrm{~g}$ via a peripherally inserted central catheter (PICC) on day 1), oxaliplatin $(50 \mathrm{mg}$ via PICC, on days 1 and 2), nimotuzumab (200 $\mathrm{mg}$ via PICC on day 1), recombinant human interleukin-2 (IL-2; 500,000 units via subcutaneous injection, twice a day on days 5-12) and granulocyte-macrophage colony-stimulating factor (GM-CSF;

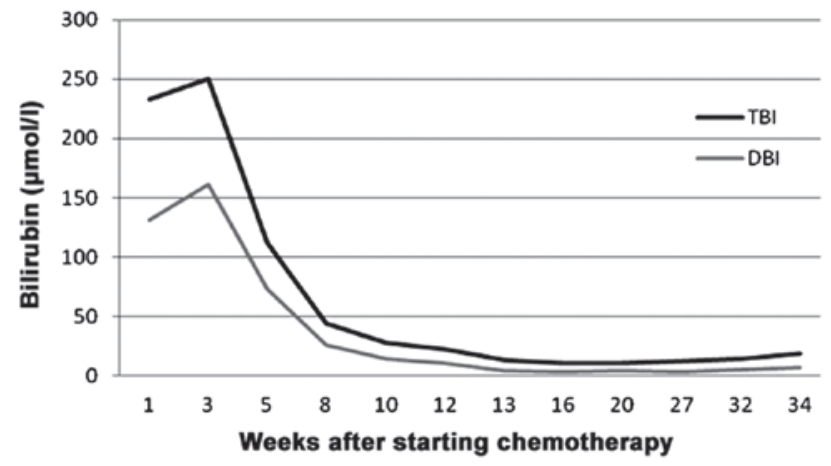

Figure 4. Graph showing total bilirubin (TBI) and direct bilirubin (DBI) levels, which decreased significantly after chemotherapy treatment.

$75 \mu \mathrm{g}$ via subcutaneous injection, twice a day on days 5-12). The patient's levels of TBI, DBI and tumor markers were found to have significantly decreased after 4 cycles of treatment (Figs. 4 and 5). A partial response (PR) was achieved at treatment weeks 8, 64 and 84 (Fig. 3B-F). Next, a 5-week course of radiation therapy was given to the hepatic portal vein, which consisted of delivering a total dose of $50 \mathrm{~Gy}$ in 25 fractions. This radiotherapy was administered concurrently with another chemotherapy strategy consisting of 5-fluorouracil (1.25 g via continuous infusion over $120 \mathrm{~h}$ every week) and nimotuzumab (200 mg every 2 weeks). The patient attained 

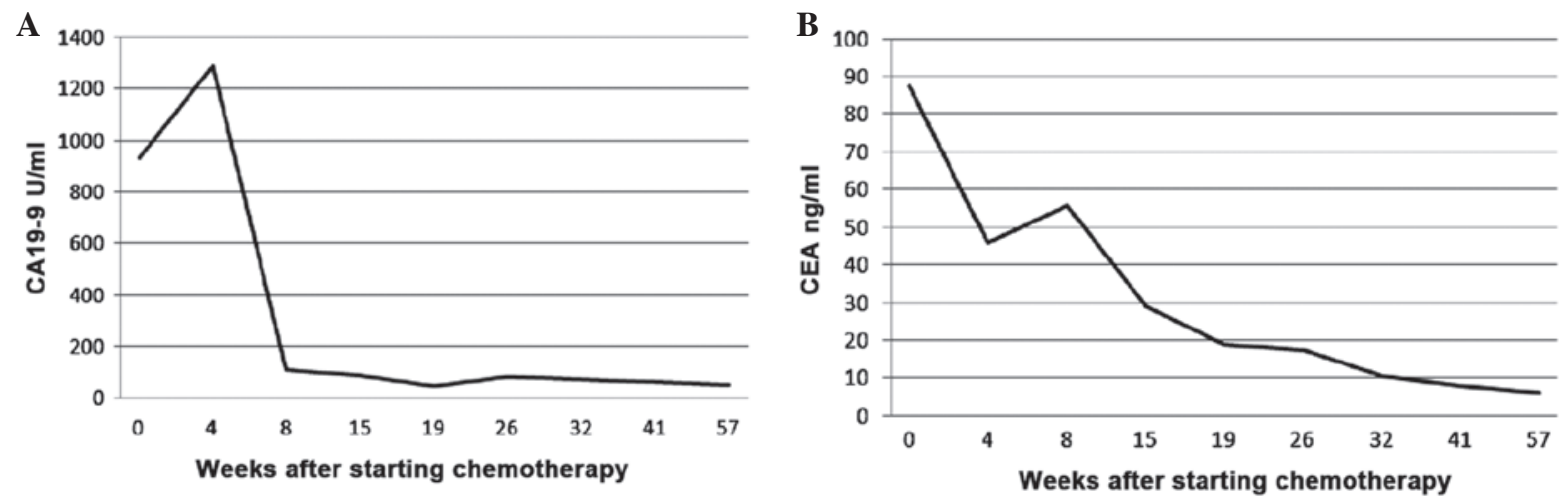

Figure 5. Graphs showing tumor marker levels. (A) The cancer antigen 19-9 (CA19-9) level returned to normal at 8 weeks post-chemotherapy treatment. (B) The carcinoembronic antigen (CEA) level gradually decreased after the administration of chemotherapy.

a complete response (CR) after completion of the chemoradiation regimen, when surgery was performed to restore the biliary duct drainage by inserting a biliary stent. A sustaining therapy regimen of compound tegafur tablets (324 mg, twice a day, on days 1-14, every 21 days) was established with the aim of assisting the patient in attaining longer-term maintenance of the CR status. The patient tolerated all the treatments well, with only mild liver dysfunction and grade 2 myelosuppression experienced.

At 3 months after being discharged, the patient was readmitted to the hospital with recurrent icteric sclera, yellowish discoloration of the urine and a fever. Laboratory tests showed elevated levels of TBI $(125.9 \mu \mathrm{mol} / \mathrm{l})$, DBI $(91.6 \mu \mathrm{mol} / \mathrm{l})$ and CA19-9 (1,349.0 U/ml); however, CEA levels were observed to be within the normal range. CT imaging revealed gas aggregation in the intrahepatic bile duct and fluid accumulation around the liver. Blood culture indicated E. coli infection, which may have been associated with the biliary stent implantation, and the patient was subsequently treated with PTCD and imipenem ( $0.5 \mathrm{~g}$, intravenous drop, every 8 hours). However, the patient did not survive the infection and succumbed 26 months after the initial diagnosis of GBC. The patient's family consented to the publication of patient data in the present case report.

\section{Discussion}

GBC is commonly diagnosed at a late stage, not only due to the aggressiveness of the disease, but also as the clinical presentation of GBC mimics that of cholelithiasis or chronic cholecystitis, both of which progress gradually over a long period of time (9). Hence, there is a possibility of missing the cancer diagnosis, an example of which is presented in the current study. Extensive IN was found during a pathological review that was conducted 2 years after the first resection sample was taken from the patient. In a retrospective review of 435 cases performed by researchers at the Memorial Sloan-Kettering Cancer Center, the incidental diagnosis of GBC during laparoscopic cholecystectomy occurred in $47 \%$ of the cases examined (6). Considering the prevalence of incidental GBC and the fact that cholelithiasis accompanied by chronic inflammation is the most well-established risk factor for GBC (10), careful histopathological review should be performed following surgery for cholelithiasis. Additionally, physicians should be aware of occult GBC in patients with other high-risk factors, including calcification of the gallbladder, anomalous pancreaticobiliary duct junctions and gallbladder polyps.

The 2000 International Agency of Research on Cancer (11) yielded a consensus on the naming of gastrointestinal precancerous lesions; the precancerous and early-stage cancers, which had been previously described as 'high-grade dysplasia', 'carcinoma in situ', 'intramucosal carcinoma' and 'local canceration', were recommended to be renamed using the more encompassing descriptor, 'intraepithelial neoplasia'. However, the understanding of IN is not yet uniform among scholars in Japan and Western countries. The former argue that IN belongs to the entity of carcinoma (according to its cytohistological features), while the latter argue that cancer should be diagnosed only when metastasis is confirmed. Considering the case presented here, we support the former, and are concerned with the possibility of missing the diagnosis of GBC.

Tumor staging is the most critical prognostic factor for GBC patients (6). In a retrospective study of 2,574 GBC cases from hospital cancer registries across the United States, the 5-year survival of stage $0, \mathrm{I}$ and II diseases was reported to be 60,39 and $15 \%$, respectively (3). However, survival rates were found to have markedly decreased in patients diagnosed with stage III and IV cancers (to 5 and $1 \%$, respectively). The AJCC (7th edition, 2010) (8) suggest that T4 tumors (a tumor invading the main portal vein or hepatic artery, or invading two or more extrahepatic organs or structures) with lymph node metastasis to the periaortic, pericaval, superior mesenteric and/or celiac arteries (N2) should be classified as stage IV disease; this classification implies that curative surgical resection should not be performed. The patient in the present study was classified as T4N2M1, and was therefore not a candidate for complete resection. The overall survival time was predicted to be $\sim 8$ months upon consideration of findings from a previous meta-analysis (4). Furthermore, the AJCC strongly recommends a second curative surgery for incidental GBC ( $\geq \mathrm{T} 1 \mathrm{~b}$ ), where the malignancy is found during or after cholecystectomy for benign gallbladder diseases. The extent of the surgery should be determined according to the stage of the cancer. The present study patient had lost the opportunity to have this second procedure performed after cholecystectomy 
due to the original missed diagnosis that had occurred years previously at a different institution. The 5-year survival for incidental GBC has been reported to be as low as $20 \%$ when a second surgery is not performed. Conversely, the 5-year survival rate has been shown to increase to $80 \%$ when patients undergo this second procedure (12). We believe that the patient presented in this case report is an example of diagnostic negligence. Better clinical outcomes would almost certainly have been achieved with correct staging and appropriate medical treatment administered after the first cholecystectomy.

The NCCN recommends biliary drainage, including a percutaneous approach (PTCD) or using an endoscopic technique such as endoscopic retrograde cholangiopancreatography, for patients presenting with jaundice (7). These procedures can result in pain relief and improved quality of life. However, it is important to note that the treatment should be performed according to the patient's condition. Treatment options for patients diagnosed with advanced-stage GBC include: i) Enrolling in a clinical trial; ii) gemcitabine- or fluoropyrimidine-based chemotherapy; and iii) best supportive care as per the NCCN guidelines (7). These recommendations were followed in the present study, and multidisciplinary collaboration was introduced into the management plan for the patient.

The rapid progression of advanced GBC provides justification for the use of adjuvant therapy, with the efficacy of chemotherapy being well established (4,13-17). Single-agent and combination regimens are applied in current clinical practice. Ueno et al (15) reported an overall response rate of $21.1 \%$ using the single chemotherapeutic agent oral fluoropyrimidine derivative $\mathrm{S}-1$. This phase II study also observed a progression-free survival (PFS) time of 3.7 months and a median overall survival time of 8.3 months. Furthermore, gemcitabine has been found to be able to achieve a longer overall survival time than any of the best supportive care strategies examined ( 9.1 vs. 2.9 months, respectively), yielding a disease control rate of $69.2 \%$ at the 1-year post-chemotherapy follow-up (14). In a study that used a pooled analysis of 104 trials, consisting of 2,810 patients with advanced biliary tract carcinoma, the combination of a gemcitabine- and a platinum-based regimen demonstrated the highest response and tumor control rates as compared with other regimens, including fluoropyrimidines plus platinum compounds, gemcitabine alone and docetaxel/paclitaxel (4). The results of a phase II trial assessing the efficacy of gemcitabine combined with carboplatin in 48 cases of advanced biliary tract carcinomas (represented by 35 cholangiocarcinoma, 12 gallbladder cancer and 1 ampullary cancer) illustrated that the median PFS time, overall survival time and 6-month survival rate were 7.8 months, 10.6 months and $85.4 \%$, respectively (17). Finally, another phase II study demonstrated that gemcitabine and oxaliplatin were associated with superior response rates (26-50\%), time-to-progression (6.5-10 months) and overall survival $(11-14$ months) $(13,16)$. Consideration of the aforementioned results and NCCN recommendations led to the choice of the combination of gemcitabine and oxaliplatin for treatment of the present study patient.

Chemoradiation aims to relieve symptoms and prolong the survival time of patients with advanced GBC. Petera et al (18) emphasized the importance of intensity-modulated radiotherapy in a study of patients with inoperable GBC or cholangiocarcinoma who were treated with a dose of 50-60 Gy/25 fractions; this treatment achieved a median overall survival time of 10.4 months. The NCCN guidelines (7) recommend concurrent chemoradiation as well, but advise that the therapeutic agents applied should be limited to either fluorouracil or capecitabine. In a retrospective study of 23 patients with non-metastatic bile duct carcinoma who underwent three-dimensional conformal external beam radiotherapy (total dose, $50.4 \mathrm{~Gy}$ ) in combination with a 5-fluorouracil-based chemotherapy after curative resection (4 gallbladder, 7 ampullary and 12 cholangiocarcinomas), the 5 -year local-regional control rate and the overall survival rate were 48.3 and $35.9 \%$, respectively (19). Those patients who had achieved negative margins after the therapy were shown to have attained better local control and overall survival rates than their counterparts with positive/narrow/unknown margins ( 67.0 vs. $35.9 \%$ and 61.4 vs. $16.7 \%$, respectively). In the present case, chemoradiation was applied following chemotherapy, which significantly improved the treatment efficacy and the patient's quality of life.

Even though various cytotoxic chemotherapeutic combinations and chemoradiation are utilized to treat advanced GBC, the PFS and overall survival times remain short. Currently, there is an increasing interest in improving the knowledge of the molecular pathways involved in carcinogenesis. Among these pathways, the epidermal growth factor receptor (EGFR) axis is believed to be one of the most important pathways involved in biliary cancers (20). Cetuximab is a promising EGFR inhibitor and has been demonstrated to be effective in a phase II trial (21). In this trial, Cetuximab, in combination with chemotherapy, was employed in unresectable local-regional or metastatic biliary cancers. In total $63 \%$ of the patients achieved an objective response, and 30\% underwent a potentially curative secondary resection after a major response to therapy. In another study investigating cetuximab-containing therapy for the treatment of 5 advanced biliary cancer patients, a CR was achieved in 1 patient, a PR in 3 patients and stable disease (SD) in 1 patient (22). Notably, a CR was observed in the patient who was wild-type for the KRAS gene. Additionally, the corresponding K-Ras protein was expressed at normal levels. Thus, a KRAS gene analysis was performed for the patient reported in the present to determine whether a mutation was present at this locus. A genetic mutation in KRAS can lead to abnormal protein function, which could affect how a patient responds to anti-EGFR therapy. Since the KRAS gene was wild-type in the patient, nimotuzumab was applied.

It has also been indicated that chemotherapy is able to cause 'inflammatory changes' in the tumor microenvironment, such as upregulation of chemokine expression, exposure of tumor antigens, prevention of immunocyte eradication and inhibition of vascular structural changes. These changes aid in enhancing the antineoplastic affect at the tumor site (23). In a previous study combining gemcitabine, an oxaliplatin, fluorouracil and folinic acid regimen, and GM-CSF/IL-2, patients with colorectal carcinoma demonstrated a high tolerance to the treatment and a favorable objective response rate (68.9\%) (24). The results of a phase II multicenter trial of maintenance biotherapy using IL-2/GM-CSF/interferon $\alpha-2 b$ following 
induction chemotherapy revealed superior CR, PR and SD rates (8, 36 and $29 \%$ respectively). The PFS time was extended to 9 months, and overall survival time was 13.5 months (25). In the present case, GM-CSF/IL-2 treatment was commenced $48 \mathrm{~h}$ after chemotherapy, as immunotherapy can activate tumor antigen-specific cytotoxic $\mathrm{T}$ lymphocyte aggregation at the tumor site, which may enhance antitumor efficacy.

In summary, at the time of admission, the patient presented with local invasion of the liver, peritoneum and lymph nodes. Cancer staging as cT4N2M1, anatomical stage IVB was clear, and multidisciplinary treatment, including PTCD, chemotherapy, chemoradiation, targeted therapy and immunotherapy, were applied. The therapeutic approach was successful, and the patient tolerated the treatment well. The overall survival of the patient was extended to 26 months with a greatly improved quality of life. We recommend that a multidisciplinary collaborative approach be integral to the management of GBC, with individual situations taken into consideration when interpreting consensus guidelines.

\section{References}

1. Randi G, Malvezzi M, Levi F, Ferlay J, Negri E, Franceschi S and La Vecchia C: Epidemiology of biliary tract cancers: An update. Ann Oncol 20: 146-159, 2009.

2. Hezel AF and Zhu AX: Systemic therapy for biliary tract cancers. Oncologist 13: 415-423, 2008.

3. Donohue JH, Stewart AK and Menck HR: The national cancer data base report on carcinoma of the gallbladder, 1989-1995. Cancer 83: 2618-2628, 1998.

4. Eckel F and Schmid RM: Chemotherapy in advanced biliary tract carcinoma: A pooled analysis of clinical trials. Br J Cancer 96 896-902, 2007.

5. Watanabe Y, Goto H, Hirooka Y, Itoh A, Taki T, Hayakawa S, Hayakawa T, Naitoh Y, Ohhashi K, Yamao K and Furukawa T: Transpapillary biopsy in gallbladder disease. Gastrointest Endosc 51: 76-79, 2000.

6. Duffy A, Capanu M, Abou-Alfa GK, Huitzil D, Jarnagin W, Fong Y, D'Angelica M, Dematteo RP, Blumgart LH and O'Reilly EM: Gallbladder cancer (GBC): 10-year experience at memorial Sloan-Kettering cancer centre (MSKCC). J Surg Oncol 98: 485-489, 2008.

7. National Comprehensive Cancer Network: NCCN Clinical Practice Guidelines in Oncology. Hepatobiliary Cancers (version 2, 2016). https://www.nccn.org/professionals/physician gls/pdf/hepatobiliary.pdf. Accessed July 6, 2016.

8. Edge S, Byrd DR, Compton CC, Fritz AG, Greene FL and Trotti A (eds): AJCC Cancer Staging Handbook. From the AJCC Cancer Staging Manual. 7th edition. Springer, New York, 2010.

9. Lazcano-Ponce EC, Miquel JF, Muñoz N, Herrero R, Ferrecio C, Wistuba II, Alonso de Ruiz P, Aristi Urista G and Nervi F: Epidemiology and molecular pathology of gallbladder cancer. CA Cancer J Clin 51: 349-364, 2001.

10. Sheth S, Bedford A and Chopra S: Primary gallbladder cancer: Recognition of risk factors and the role of prophylactic cholecystectomy. Am J Gastroenterol 95: 1402-1410, 2000.
11. Bosman FT, Carneiro F, Hruban RH and Threise ND (eds): WHO Classification of Tumours of the Digestive System. 4th edition. IARC Press, Lyon, 2010.

12. Shimizu T, Arima Y, Yokomuro S, Yoshida H, Mamada Y, Nomura T, Taniai N, Aimoto T, Nakamura Y, Mizuguchi Y, et al: Incidental gallbladder cancer diagnosed during and after laparoscopic cholecystectomy. J Nippon Med Sch 73: 136-140, 2006.

13. Gebbia N, Verderame F, Di Leo R, Santangelo D, Cicero G, Valerio MR, Arcara C, Badalamenti G, Fulfaro F and Carreca I: A phase II study of oxaliplatin $(\mathrm{O})$ and gemcitabine $(\mathrm{G})$ first line chemotherapy in patients with advanced biliary tract cancers. J Clin Oncol 23 (suppl): abstract 4132, 2005.

14. Kuriyama H, Kawana K, Taniguchi R, Jono F, Sakai E, Okubo H, Suzuki H, Kobayashi S, Murata Y, Inamori M, et al: Single-agent gemcitabine in elderly patients with unresectable biliary tract cancer. Hepatogastroenterology 58: 26-30, 2011.

15. Ueno H, Okusaka T, Ikeda M, Takezako Y and Morizane C: Phase II study of S-1 in patients with advanced biliary tract cancer. Br J Cancer 91: 1769-1774, 2004.

16. Harder J, Riecken B, Kummer O, Lohrmann C, Otto F, Usadel H, Geissler M, Opitz O and Henss H: Outpatient chemotherapy with gemcitabine and oxaliplatin in patients with biliary tract cancer. Br J Cancer 95: 848-852, 2006.

17. Williams KJ, Picus J, Trinkhaus K, Fournier CC, Suresh R, James JS and Tan BR: Gemcitabine with carboplatin for advanced biliary tract cancers: A phase II single institution study. HPB (Oxford) 12: 418-426, 2010

18. Petera J, Kasaová L, Paluska P, Sirák I, Jansa J, Macingová Z, Dvorák J and Soumarova R: Intensity-modulated radiotherapy in the treatment of subhepatic carcinomas. Hepatogastroenterology 58: 331-335, 2011.

19. Bonet Beltrán M, Roth AD, Mentha G and Allal AS: Adjuvant radio-chemotherapy for extrahepatic biliary tract cancers. BMC Cancer 11: 267, 2011.

20. Yoshikawa D, Ojima H, Iwasaki M, Hiraoka N, Kosuge T, Kasai S, Hirohashi S and Shibata T: Clinicopathological and prognostic significance of EGFR, VEGF, and HER2 expression in cholangiocarcinoma. Br J Cancer 98: 418-425, 2008.

21. Gruenberger B, Schueller J, Heubrandtner U, Wrba F, Tamandl D Kaczirek K, Roka R, Freimann-Pircher S and Gruenberger T: Cetuximab, gemcitabine, and oxaliplatin in patients with unresectable advanced or metastatic biliary tract cancer: A phase 2 study. Lancet Oncol 11: 1142-1148, 2010.

22. Chang PY, Cheng MF,Lee HS, Hsieh CB and Yao NS: Preliminary experience of cetuximab in the treatment of advanced-stage biliary tract cancer. Onkologie 33: 45-47, 2010.

23. Zitvogel L, Apetoh L, Ghiringhelli F, André F, Tesniere A and Kroemer G: The anticancer immune response: Indispensable for therapeutic success? J Clin Invest 118: 1991-2001, 2008

24. Correale P, Cusi MG, Tsang KY, Del Vecchio MT, Marsili S, Placa ML, Intrivici C, Aquino A, Micheli L, Nencini C, et al: Chemo-immunotherapy of metastatic colorectal carcinoma with gemcitabine plus FOLFOX 4 followed by subcutaneous granulocyte macrophage colony-stimulating factor and interleukin-2 induces strong immunologic and antitumor activity in metastatic colon cancer patients. J Clin Oncol 23: 8950-8958, 2005.

25. O'Day SJ, Atkins MB, Boasberg P, Wang HJ, Thompson JA, Anderson CM, Gonzalez R, Lutzky J, Amatruda T, Hersh EM and Weber JS: Phase II multicenter trial of maintenance biotherapy after induction concurrent biochemotherapy for patients with metastatic melanoma. J Clin Oncol 27: 6207-6212, 2009. 